\title{
Successful Recovery from an Acute Kidney Injury due to Amniotic Fluid Embolism
}

\author{
Katsuhito Ihara ${ }^{1}$, Shotaro Naito ${ }^{1}$, Tomokazu Okado ${ }^{2}$, Tatemitu Rai ${ }^{2}$, Yutaro Mori ${ }^{1}$, \\ Takayuki Toda ${ }^{1}$, Shinichi Uchida ${ }^{2}$, Sei Sasaki ${ }^{2}$ and Noriaki Matsui ${ }^{1}$
}

\begin{abstract}
A 33-year-old Japanese woman at 40 weeks gestation visited the maternity hospital after imminent labor had begun. After the delivery, persistent bleeding developed resulting in hemorrhagic shock. Although the hemorrhage was eventually controlled, hepatic and renal dysfunction occurred, leading to acute kidney injury (AKI). The patient's clinical presentation was suggestive of amniotic fluid embolism (AFE). We subsequently initiated continuous renal replacement therapy (RRT) for AKI. The patient's condition improved, she discontinued RRT, and her renal function recovered. We herein report a patient who successfully recovered from AKI caused by AFE.
\end{abstract}

Key words: acute kidney injury, amniotic fluid embolism, multiple organ failure, renal replacement therapy, acute tubular necrosis, bilateral renal cortical necrosis

(Intern Med 54: 49-54, 2015)

(DOI: 10.2169/internalmedicine.54.2348)

\section{Introduction}

Amniotic fluid embolism (AFE) is a rare but life-threating condition that occurs either during pregnancy or shortly after delivery. A recent study indicated that the incidence of AFE ranges between 1.9 and 6.1 cases per 100,000 maternities (1). AFE has a high mortality rate ranging from 13.3$40.0 \%$ (2) with an unexpected prevalence of respiratory and circulatory failure and disseminated intravascular coagulation (DIC). The causative agents of AFE are considered to be present in the meconium. Chemical transmitters in the amniotic fluid, such as prostaglandins, kinins, and serotonin, as well as secondary messengers from the lung tissues released by vascular occlusion are associated with the pathogenesis of AFE. Major clinical consequences of AFE are anaphylaxis, vascular occlusion, vasoconstriction mediated by prostaglandins, and DIC (2). The risk factors for AFE include maternal age (particularly $>35$ years), parity, smoking, race, diabetes, multiple pregnancy, previous cesarean delivery, placenta previa, placental abruption, labor induction, mode of delivery, and manual removal of placenta (1). A typical clinical scheme of AFE starts with shock after acute dyspnea and chest pain among expectant and nursing mothers without any complications at the latter part of the first stage of labor or immediately after labor. Following shock, patients are prone to bleeding due to DIC, with large amounts of genital bleeding, which can lead to death. AFE is clinically diagnosed on the basis of nonspecific clinical symptoms, such as the sudden onset of acute cardiorespiratory failure with DIC from an unknown cause. Other causes of sudden intrapartum or postpartum cardiorespiratory failure must be excluded (3). Recently, serological diagnostic methods that may facilitate the diagnosis of AFE have been developed, including measurements of coproporphyrins in the fetal urine, zinc coproporphyrins in the meconium, and sialyl Tn (STN) antigen in the maternal blood (4). Acute kidney injury (AKI) may occur as a consequence of hypotension and DIC in AFE. Bilateral renal cortical necrosis (BRCN) may also develop as a result of hypotension and DIC. We herein report a patient with AKI due to AFE who successfully recovered and her renal function subsequently improved.

${ }^{1}$ Department of Nephrology, Tsuchiura Kyodo General Hospital, Japan and ${ }^{2}$ Department of Nephrology, Tokyo Medical and Dental University, Japan

Received for publication December 19, 2013; Accepted for publication May 12, 2014

Correspondence to Dr. Katsuhito Ihara, ctngt512@yahoo.co.jp 

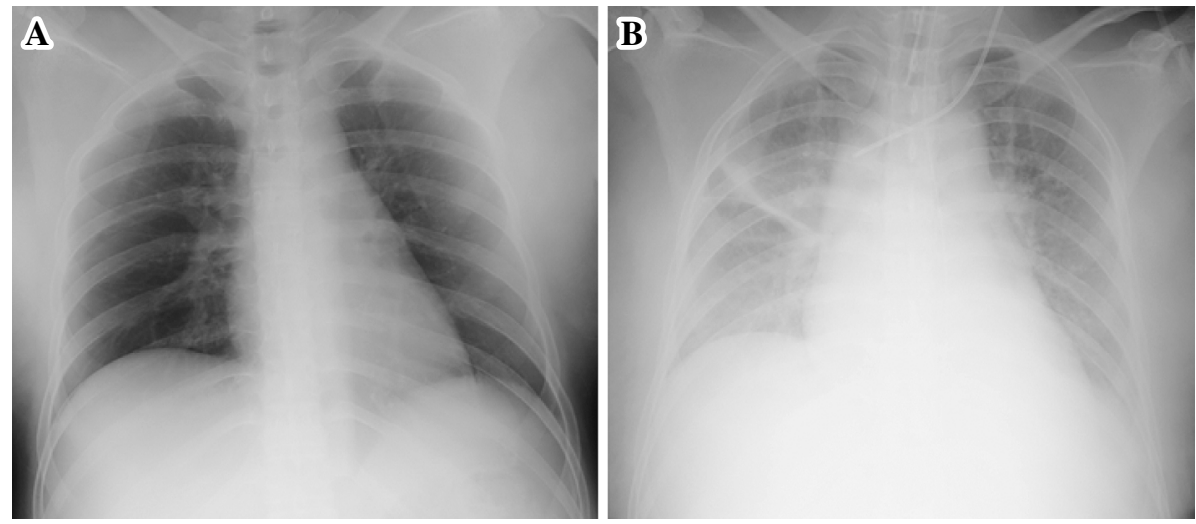

Figure 1. (A) Chest radiograph on Day 1. Bilateral lung congestion had not yet appeared. (B) Chest radiograph on Day 3. Bilateral lung congestion gradually progressed, as observed by chest radiography, until the day of the consultation.

\section{Case Report}

A 33-year-old Japanese woman at 40 weeks gestation was admitted to the maternity hospital due to syncope. Her syncope soon disappeared, and labor was initiated. Forced delivery and precipitous labor were performed in an hour. After delivery, bleeding from the perineum and vaginal wall persisted despite suturing the wound. Because the total amount of hemorrhage reached $2,700 \mathrm{~mL}$ and the patient's $\mathrm{Hb}$ values declined to $3.6 \mathrm{~g} / \mathrm{dL}$, she was transferred to the emergency department of our hospital. An initial diagnosis of atonic bleeding was confirmed, and bimanual pressure hemostasis was conducted. However, the bleeding could not be stopped, and the patient went into a coma, hemorrhagic shock, acute respiratory failure with immeasurable oxygen saturation, and DIC. Head computed tomography (CT) was performed to assess the disturbance of consciousness with unremarkable findings. Enhanced CT on admission suggested ahemorrhage from the left vaginal wall. She was intubated and admitted to the intensive care unit (ICU). The Sequential Organ Failure Assessment score was 10 points on admission to ICU. Cefmetazon (CMZ) was administered. Red cell concentrate (RCC), fresh frozen plasma (FFP), platelet-rich plasma, gabexate mesilate, urinastatin, human anti-thrombin III, and Eptacog alfa (a recombinant human factor VIIa) were administered to treat DIC. Because the vaginal wall bleeding could not be controlled, angiography was performed. The hemorrhage was finally controlled through the obstruction of the left internal iliac artery by acoil insertion. However, hepatic and renal dysfunction occurred the next day leading to multiple organ failure (MOF). The patient's serum creatinine ( $\mathrm{SCr}$ ) levels increased from 0.78 to $4.35 \mathrm{mg} / \mathrm{dL}$ in 48 hours, which met the definition of AKI. The AKI stage in this case was categorized as "Failure" according to the Risk, Injury, Failure; Loss, End-Stage Renal Disease criteria and stage III according to the Acute Kidney Injury Network criteria. In addition, hepatic failure was observed, and the patient's aspartate aminotransferase, alanine aminotransferase, alkaline phosphatase, lactate dehy- drogenase, and total bilirubin levels were 4,079 IU/L, 1,685 $\mathrm{IU} / \mathrm{L}, 457 \mathrm{IU} / \mathrm{L}, 5,569 \mathrm{IU} / \mathrm{L}$, and $2.4 \mathrm{mg} / \mathrm{dL}$, respectively. An increased level of creatine kinase (13,338 IU/L) was observed; myoglobinuria was considered as a possible cause of AKI. In the patient's urinalysis, hematuria was positive by the dipstick method (3+), and the urinary sediment was positive, showing a red blood cell count of $\geq 100 / \mathrm{hpf}$. Because the urinary sediment was positive, myoglobinuria was ruled out. Extremely high levels of zinc coproporphyrin 1 (6.2 $\mathrm{pmol} / \mathrm{mL}$; normal range $<1.6 \mathrm{pmol} / \mathrm{mL}$ ) and STN antigen $(471.3 \mathrm{U} / \mathrm{mL}$; normal range $<45.0 \mathrm{U} / \mathrm{mL})$ were detected in the maternal blood. Although hemorrhagic shock was strongly associated with this patient's hemodynamic state, a diagnosis of AFE was confirmed on the basis of the severe clinical course of precipitous labor and cardiovascular collapse after delivery, and AFE was considered the cause of DIC and MOF. The platelet count, prothrombin time (PT), activated partial thromboplastin time (APTT), fibrinogen, and fibrin/fibrinogen degradation product were $9.4 \times 10^{4} / \mu \mathrm{L}$, $\leq 5 \%, \geq 240 \mathrm{~s}$, immeasurable, and $362.0 \mu \mathrm{g} / \mathrm{mL}$, respectively. When we considered AFE to be the underlying cause of DIC, the obstetrical DIC score was 23 points on admission.

The patient did not have a significant medical history. Her father had hypertension. She did not smoke, drink alcohol, or take any medications. She had no known allergies. Upon examination on the day of consultation, her height was 154 $\mathrm{cm}$ and her body weight was $80.0 \mathrm{~kg}$ (prenatal body weight, $62.0 \mathrm{~kg}$ ). The patient's vital signs and oxygen saturation were stable, and the following measurements were recorded: body temperature, $36.8^{\circ} \mathrm{C}$; blood pressure, $104 / 72 \mathrm{mmHg}$; heart rate, 109 beats/min and regular; respiratory rate, 19 breaths/min; oxygen saturation, $100 \%$ on mechanical ventilation [continuous positive airway pressure and pressure support (PS) mode; $\mathrm{FiO}_{2} 0.5$, PS of $8 \mathrm{~cm} \mathrm{H}_{2} \mathrm{O}$, positive endexpiratory pressure of $10 \mathrm{~cm} \mathrm{H}_{2} \mathrm{O}$ ]. No heart murmur was audible. She had attenuating respiratory sounds in both her lungs. Bilateral lung congestion gradually progressed, as observed by chest radiography, until the day of consultation (Fig. 1). Bilateral leg edema was present.

On day 3 (the day of consultation), the patient's platelet 


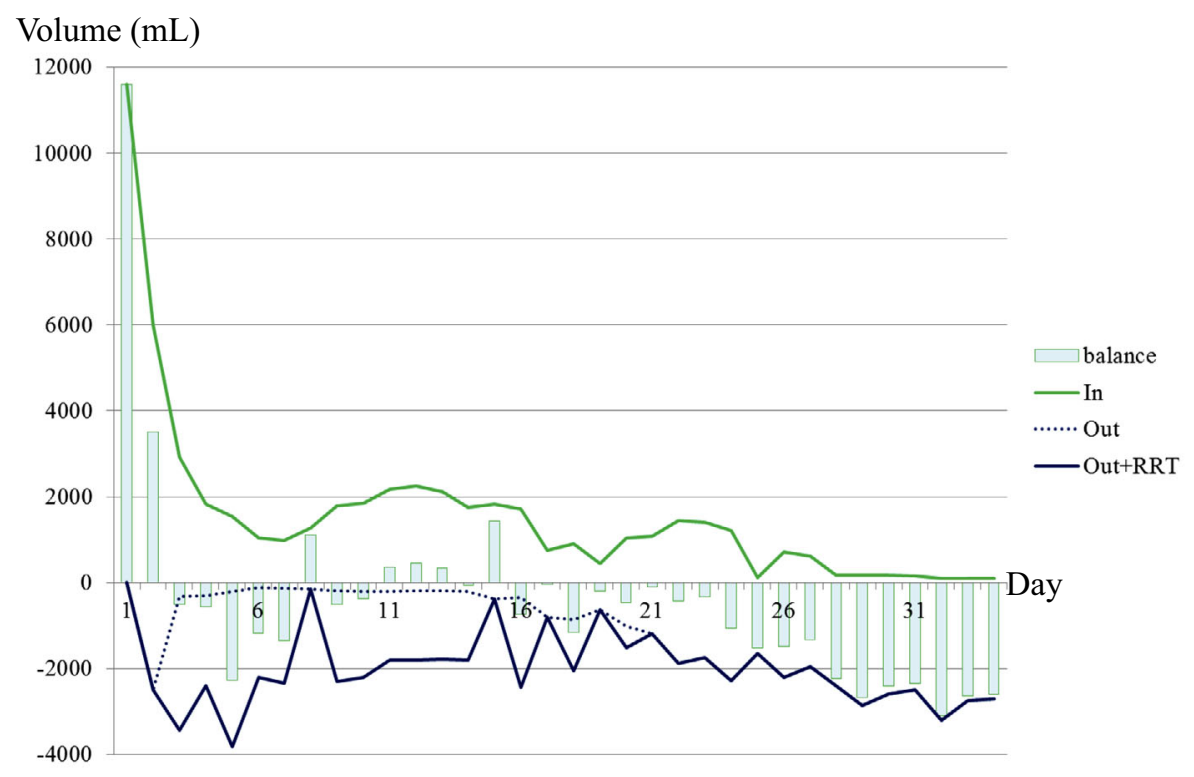

Figure 2. The water balance after admission. Intake indicates the intravenous and enteral nutrition. Output indicates the urinary volume and removal of water by renal replacement therapy (RRT). Water ingestion is not shown.

count was $5.5 \times 10^{4} / \mu \mathrm{L}$ after platelet transfusion and DIC treatment, $\mathrm{Hb}$ levels were $8.0 \mathrm{~g} / \mathrm{dL}$, and the hematocrit value was $22.6 \%$ after RCC transfusion. The PT recovered from $\leq$ 5 to $30 \%$, and the APTT declined from $\geq 240$ to 42.8 s, suggesting an improvement of DIC. The fractional excretion of sodium (FENa) was $7.70 \%$, and the sodium excretion in the urine was $132 \mathrm{mEq} / \mathrm{day}$ and $53.1 \mathrm{mEq} / \mathrm{L}$ on day 2 . These results suggested that AKI was caused by renal injury. We decided to initiate renal replacement therapy (RRT) for oliguria and hypervolemia due to AKI on day 3. As the patient's blood pressure was unstable, we selected continuous hemodiafiltration (CHDF) as the treatment modality of RRT. In our hospital, we could not continue continuous renal replacement therapy (CRRT) overnight due to the lack of clinical engineering staff at night. We conducted CHDF as the modality of CRRT with diafiltrate and replacement fluid: Subpack-Bi ${ }^{\circledR}$ (Nipro Co., Ltd., Osaka, Japan) hemodialyzer: cellulose triacetate (CTA) $1.1 \mathrm{~m}^{2}$ [UT-1100S ${ }^{\circledR}$ (Nipro Co., Ltd.)], blood flow rate $(\mathrm{QB}): 100 \mathrm{~mL} / \mathrm{min}$, dialysate flow rate $(\mathrm{QD})+$ filtration flow rate $(\mathrm{QF}): 900-1,070 \mathrm{~mL} / \mathrm{hr}$, replacement flow rate (QS): $400-530 \mathrm{~mL} / \mathrm{hr}$, and anticoagulant: nafamostat mesilate $(30 \mathrm{mg} / \mathrm{hr})$. The activated clotting time of the blood circuit was frequently monitored to minimize the risk of additional bleeding by the anticoagulant. By removing the excess water through $\mathrm{CHDF}$, the patient's congestive heart failure was controlled, which led to a wellbalanced volume status over time (Fig. 2). The patient resumed spontaneous respiration as the congestion was alleviated. Although we attempted to wean her from ventilator support, she did not easily wake up possibly due to the accumulation of sedative drugs. We had administered midazolam, a benzodiazepine, to keep the depth of sedation while targeting a score of -4 on the Richmond Agitation-Sedation Scale. Midazolam has a half-life of 3-11 hours, and the ac- tive metabolite accumulates with prolonged infusion, leading to the necessity of removal of the drug (5). Furthermore, because ventilator-associated pneumonitis may have contributed to the patient's condition, as assessed by her respiratory condition and clinical progress, the antibiotic drug was changed from CMZ to piperacillin/tazobactam (PIPC/TAZ). We altered the modality of RRT to intermittent hemodialysis on day 9 , after removing the sedative drugs. On days 9 and 10 , we performed hemodialysis for 4 hours with dialyzer: polymethylmethacrylate $1.0 \mathrm{~m}^{2}\left[\mathrm{BK}-1.0 \mathrm{~F}^{\mathbb{Q}}\right.$ (Toray Medical, Co., Ltd., Chiba, Japan)], QB: $120 \mathrm{~mL} / \mathrm{min}$, and QD: 300 $\mathrm{mL} / \mathrm{min}$. From day 11, we performed HD for 4 hours with dialyzer: CTA $1.5 \mathrm{~m}^{2}$ [FB-150 U $\beta^{\circledR}$ (Nipro Co., Ltd.)], QB: $150 \mathrm{~mL} / \mathrm{min}$, and QD: $450 \mathrm{~mL} / \mathrm{min}$. As a result, her consciousness and respiratory condition improved with successful weaning from mechanical ventilation. She was finally extubated on day 14 with appropriate spontaneous respiration. Her urine volume substantially increased from day 17, with stable hemodynamics, which led to the improvement of her renal function. We successfully withdrew RRT on day 20, and her renal function remained stable (Fig. 3). She recovered and significant rehabilitation occurred in the general ward. She was discharged on day 35 , and she is currently visiting our internal outpatient clinic to monitor her renal function.

\section{Discussion}

When amniotic fluid flows into the maternal blood, chemical mediators are released in response to the stimulation of fetal remnants in the amniotic fluid, causing DIC as a result of protease activation. Fetal remnants and chemical mediators trigger hypercytokinemia, causing shock and systemic inflammatory response syndrome (SIRS), thereby 


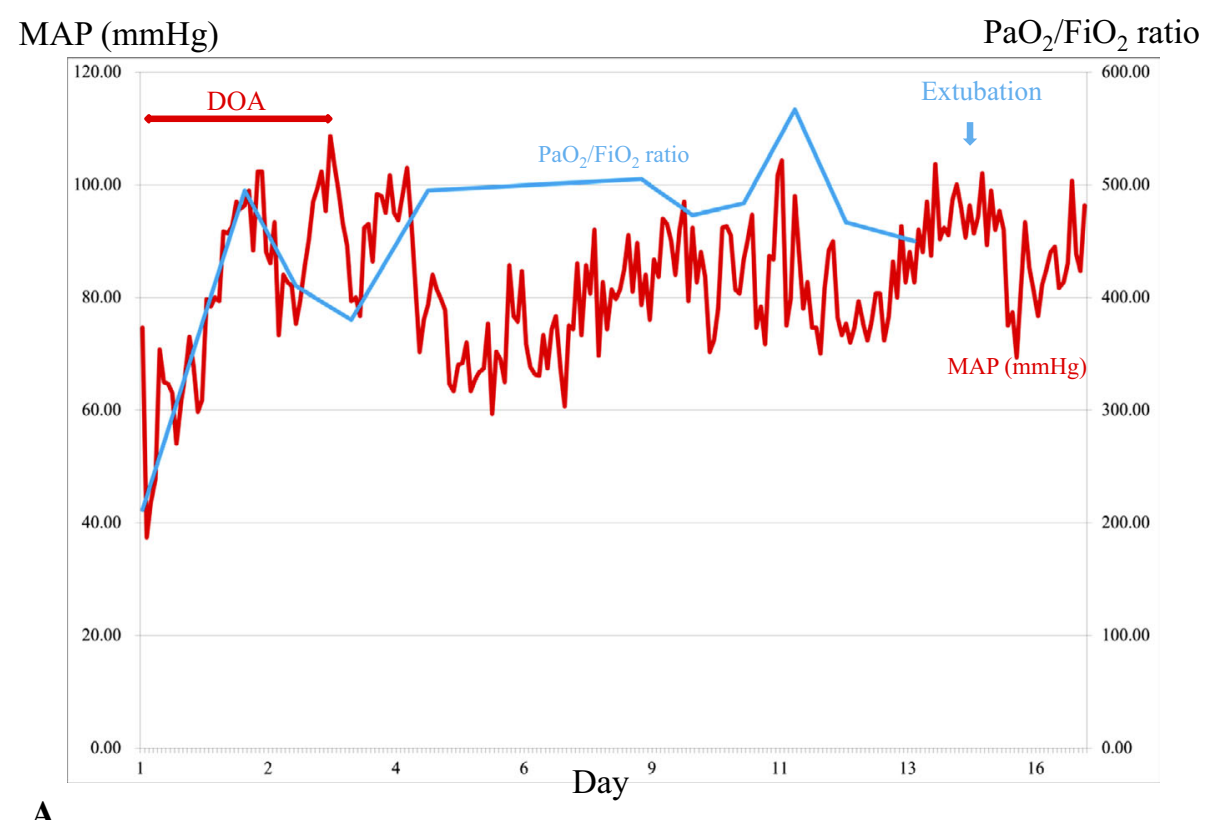

$\mathbf{A}$

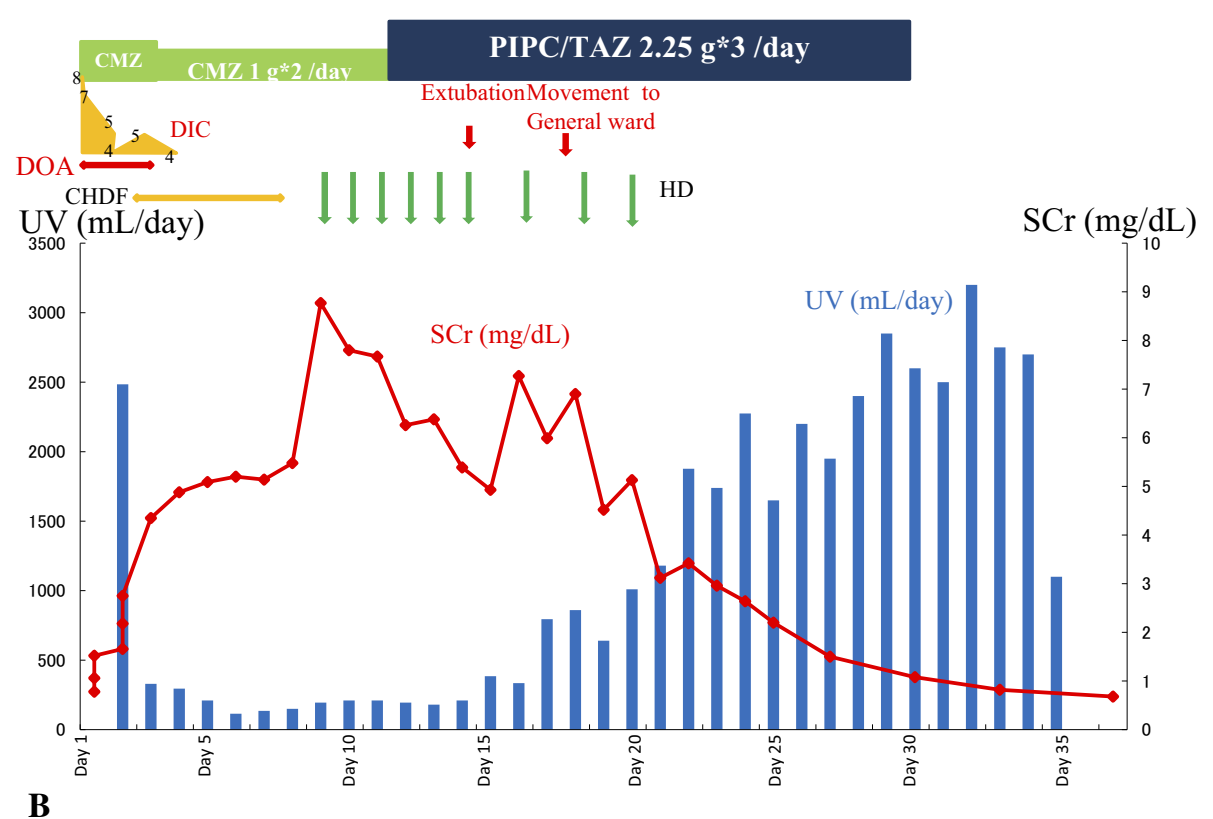

Figure 3. (A) The mean arterial blood pressure and the $\mathrm{PaO}_{2} / \mathrm{FiO}_{2}$ ratio. These parameters improved daily. (B) The patient's urinary volume substantially increased from day 17 , leading to the improvement of her renal function. Successful withdrawal of renal replacement therapy (RRT) was achieved on day 20, and the patient's renal function remained stable. AKI: acute kidney injury, AFE: amniotic fluid embolism, CMZ: cefmetazon, PIPC/TAZ: piperacillin/tazobactam, DIC: disseminated intravascular coagulation, DOA: dopamine, UV: urine volume, $\mathrm{mL} / \mathrm{d}: \mathrm{mL} / \mathrm{day}$, SCr: serum creatinine, CHDF: continuous hemodiafiltration, HD: hemodialysis

leading to the development of renal failure and MOF (6). The major clinical findings of AFE include the abrupt and fulminant onset of hypotension due to cardiogenic shock, hypoxemia and respiratory failure, DIC, and coma or seizures (3).

We herein presented a case of a 33-year-old woman with massive hemorrhaging and cardiorespiratory failure due to AFE immediately after delivery. AFE is essentially a diagnosis based on the clinical findings. Our patient received manual removal of the placenta, which is one of the risk factors for AFE. In addition, our patient had high levels of zinc coproporphyrin 1 and STN antigen, which are normally absent in the maternal blood. Although there are no reports referring to the sensitivity and specificity of zinc coproporphyrin 1 and STN antigen in AFE, these serological markers are considered useful in the diagnosis of AFE (7). Oi et al. reported that the mean serum concentration of STN in 19 patients with AFE was $110.8 \mathrm{U} / \mathrm{mL}$, which was significantly 
higher when compared with patients without AFE (17.3 U/ $\mathrm{mL}$ ) (8). Our patient's STN concentration was $471.3 \mathrm{U} / \mathrm{mL}$. Based on these findings, we diagnosed our patient with AFE rather than hemorrhagic shock.

Our patient had severe DIC due to AFE. In most cases of AFE, consumption of coagulation factors, particularly fibrinogen, by the amniotic fluid and fetal remnants is the first pathophysiological step of DIC compared with the other underlying causes of DIC in which vascular endothelial dysfunction is the initiating event. Indeed, in our patient, the value of fibrinogen was immeasurable, thus suggesting early consumption of the coagulation factors. Therefore, an early measurement of fibrinogen is recommended to diagnose AFE.

In general, AKI secondary to AFE is caused by ischemic consequences of hypotension following cardiac shock, hypoxic brain injury, myocardial injury, and DIC (3). BRCN may pathophysiologically develop as a result of severe hypotension and DIC associated with AFE. In less severe cases of hypotension and DIC, acute tubular necrosis may be induced (9). DIC has been pathophysiologically associated with glomerular microthrombosis and AKI. In the presence of inflammation, monocytes/macrophages are induced to migrate into the glomeruli where, after their activation, they produce tissue factors or procoagulant activity, leading to fibrin deposition. In addition, mesangial cells participate in glomerular fibrin deposition (10). Both severe renal ischemia and DIC leading to endothelial damage and secondary fibrin deposition have been proposed as the initiating events in AFE. When endothelial injury occurs, the local release of nitric oxide, an endothelium-derived relaxing factor, normally minimizes the degree of thrombus formation by diminishing platelet aggregation (11). Affected patients with BRCN abruptly develop oliguria or anuria, which is frequently accompanied by gross hematuria and flank pain. Anuria or oliguria can last more than 15-20 days (9). The clinical course of acute BRCN depends on the extent of involvement; if necrosis is extensive, death occurs within the first few days unless dialysis is performed. However, with timely RRT, renal function may recover sufficiently to allow patients to become dialysis independent (10). A renal biopsy may be performed; however, this invasive procedure is not required in most cases. The diagnosis is usually established by ultrasonography or CT $(12,13)$. In our patient, oliguria and gross hematuria were observed after admission, and these findings subsided after RRT. Enhanced CT revealed decreased enhancement of both kidneys indicating renal perfusion. A renal biopsy was not performed; however, the complete recovery of the patient's renal function suggested that the cause of AKI had most likely been acute renal necrosis as opposed to BRCN.

The hemodynamic support with intravenous fluid administration, sufficient oxygenation by mechanical ventilator, and improvement of pulmonary edema by RRT led to increased renal perfusion, and the patient's renal function significantly recovered. In addition, RRT has two specific roles in the treatment of AFE. One is the removal of substances associated with AFE. The primary causative substances of AFE are fetal remnants in the amniotic fluid flowing into the maternal blood. Extrinsic proteinases in the amniotic fluid and chemical mediators and cytokines released after stimulation by fetal remnants lead to shock and MOF, including DIC and AKI. In particular, glomerular microthrombosis is a causative factor for AKI induced by DIC. The specific function of RRT during AFE is the removal of fetal remnants from the maternal circulation. Consequently, the removal of substances derived from fetal remnants in the amniotic fluid may lead to the improvement of DIC and AKI. Ogihara et al. reported that CHDF enables the continuous exchange of extracellular fluid containing meconium in the amniotic fluid. Dialysis using an artificial extracellular solution may remove many substances with molecular weights (MWs) of up to $30-40 \mathrm{kDa}$. In addition, humoral factors removed by CHDF play an important role in the pathophysiology of AFE (6). The careful removal of cytokines, such as interleukin-6 (21 kDa) and interleukin-8 (8 kDa), using $\mathrm{CHDF}$ is a reasonable method for treating hypercytokinemia, which can cause MOF. Therefore, CHDF may eliminate the amniotic fluid from the maternal blood stream and metabolic acidosis produced by amniotic fluid leakage (14).

Another function of RRT in the treatment of AFE is renal protection, support, and prevention of disease aggravation. In our patient, the most important outcome was survival from AFE, and complete recovery of her renal function from AKI was considered the secondary outcome. Although we did not perform a renal biopsy in our patient, we assessed the patient's renal pathophysiology as acute renal necrosis from the result of complete renal recovery after treatment. Considering the possibility of aggravation to $\mathrm{BRCN}$, physicians should initiate RRT for renal protection and support to prevent progression to $\mathrm{BRCN}$ as early as possible when anuria or oliguria is encountered in patients with AFE. Ostermann et al. reported that RRT may be beneficial when initiated early to maintain metabolic and volume homeostasis and prevent further deterioration in patients with AKI and severe multi-organ dysfunction (15). Kaneko et al. described that only short-term CHDF may be necessary in patients with AFE because the total amount and duration of amniotic fluid leakage into the maternal blood stream is limited (14). In our patient, the actual duration of continuous RRT for maintaining the hemodynamic state and removal of substances was only 5 days, and the total duration for RRT was only 18 days until complete recovery of the patient's renal function.

In this report, we described a patient who successfully recovered from AKI caused by AFE because of the immediate initiation of RRT. To the best of our knowledge, there are no previous data regarding the frequency of AKI in AFE, although there are a few reports of AKI with AFE. Price et al. reported that recovery from AKI in AFE is usually complete without long-term sequelae if the patient overcomes embolic and coagulative problems (3). The present report suggests 
that most AKI cases associated with AFE are due to acute tubular necrosis with complete recovery of renal function. In contrast, when BRCN is induced by AFE, $20-40 \%$ patients with AFE-induced AKI displayed partial recovery with a $\mathrm{Cr}$ clearance that stabilizes between 15 and $50 \mathrm{~mL} / \mathrm{min}$ (9). An immediate initiation of RRT may completely recover renal function by preventing bilateral renal cortical necrosis.

AFE is a catastrophic event with nonspecific symptoms and carries a high mortality rate; however, rapid diagnosis and resuscitation may decrease the mortality associated with AFE (16). Our report describes a patient with AFE who successfully recovered because of the early diagnosis and supportive treatment of her cardiogenic shock, hypoxemia and respiratory failure, DIC, and AKI. We strongly recommend that RRT be immediately initiated when AFE is diagnosed.

The authors state that they have no Conflict of Interest (COI).

\section{Acknowledgement}

We wish to thank Drs. Satoshi Kimura and Naohiro Kanayama (Department of Obstetrics and Gynecology, Hamamatsu University School of Medicine, Hamamatsu, Shizuoka, Japan) for measuring the zinc coproporphyrins and STN antigen.

\section{References}

1. Knight M, Berg C, Brocklehurst $P$, et al. Amniotic fluid embolism incidence, risk factors and outcomes: a review and recommendations. BMC Pregnancy Childbirth 12: 1-11, 2012.

2. Lee JH, Yang HJ, Kim JH, et al. Aminiotic fluid embolism that took place during an emergent Cesarean section -A case report-. Korean J Anesthesiol 59: S158-S162, 2010.

3. Price TM, Baker VV, Cefalo RC. Amniotic fluid embolism. Three case reports with a review of the literature. Obstet Gynecol Surv
40: 462-475, 1985.

4. Kanayama N, Yamazaki T, Naruse Y, Sumimoto K, Horiuchi K, Terao T. Determining zinc coproporphyrin in maternal plasma -a new method for diagnosing amniotic fluid embolism. Clin Chem 38: 526-529, 1992

5. Reade MC, Finter S. Sedation and delirium in the intensive care unit. N Engl J Med 370: 444-454, 2014.

6. Ogihara T, Morimoto K, Kaneko Y. Continuous hemodiafiltration for potential amniotic fluid embolism: dramatic response observed during a 10-year period report of three cases. Ther Apher Dial 16: 195-197, 2012.

7. The Japanese Society of Obstetrical, Gynecological \& Neonatal Hematology. [Amniotic fluid embolism][Internet]. Available from: http://www.jsognh.jp/society/amniotic.php (in Japanese).

8. Oi H, Kobayashi H, Hirashima Y, et al. Serological and immunohistochemical diagnosis of amniotic fluid embolism. Semin Thromb Hemost 24: 479-484, 1998.

9. Matlin RA, Gary NE. Acute cortical necrosis: Case report and review of the literature. Am J Med 56: 110-118, 1974.

10. Gando S. Microvascular thrombosis and multiple organ dysfunction syndrome. Crit Care Med 38: S35-S42, 2010.

11. Busing CM, Bleyl U. Shock in pregnancy: pathophysiology and morphologic findings. Path Res Pract 165: 253-268, 1979.

12. Jennette JC, Olson JL, Schwartz MM, Silva FG. Renal cortical necrosis. In: Heptinstall's Pathology of the Kidney. 6th ed. Lippincott Williams \& Wilkins, Philadelphia, 2007: 1178-1179.

13. Colvin RB, Chang A, Brad Farris AB III, et al. Renal cortical necrosis. In: Diagnostic Pathology. Kidney Diseases. 1st ed. Amirsys, Salt Lake City, 2011: 4-16-4-17.

14. Kaneko Y, Ogihara T, Tajima H, Mochimaru F. Continuous hemodiafiltration intravascular coagulation and shock due to amniotic fluid embolism: report of a dramatic response. Intern Med 40: 945-947, 2001.

15. Ostermann M, Dickie H, Barrett NA. Renal replacement therapy in critically ill patients with acute kidney injury -when to start. Nephrol Dial Transplant 27: 2242-2248, 2012.

16. Aziz ES. A successfully resuscitated case of amniotic fluid embolism. Eur J Anaesthesiol 17: 712-713, 2000.

(C) 2015 The Japanese Society of Internal Medicine http://www.naika.or.jp/imonline/index.html 